\title{
Laryngeal angioleiomyoma: A case report of 52-year-old female
}

\author{
Ahmad Rezaee $^{1}$, Leili Ebrahimi ${ }^{*}$, Alireza Sadeghipour ${ }^{3}$, Behzad Sarvar Azimzadeh $^{4}$ \\ Amir Reza Azizian ${ }^{2}$ \\ ${ }^{1}$ Faculty of Medicine, Hamadan University of Medical Sciences, Hamadan, Iran \\ ${ }^{2}$ Shaheed Rajaei Cardiovascular, Medical and Research Center, Tehran University of Medical Sciences, Tehran, Iran; \\ *Corresponding Author: lilieage@gmail.com \\ ${ }^{3}$ Rasoul Akram Medical Complex, Tehran University of Medical Sciences, Tehran, Iran \\ ${ }^{4}$ Department of Cardiology, Kerman University of Medical Sciences, Kerman, Iran
}

Received 23 January 2013; revised 27 February 2013; accepted 3 March 2013

\section{ABSTRACT}

Introduction: Laryngeal angioleiomyoma is a rare benign tumor of larynx with vascular origin. We report a case of laryngeal angioleiomyoma in a 52-year-old female. Case Presentation: A 52year-old Caucasian female was referred to our institute with a chief compliant of severe midnight snoring. Her symptoms were progressive and began from two years ago. There was no history of hoarseness and laryngeal pain. The patient underwent a laryngoscopic examination and a large mass was seen on the left aryepiglotic fold. Surface of lesion was smooth and covered by laryngeal mucosa with no ulcer. There was a rapid enhancing mass on left aryepiglotic fold with bulging into the left piriformis sinus in contrast-enhanced computed tomography. The tumor was complicated by profuse bleeding after biopsy which was not controlled by conventional approaches. The patient was then administered a general anesthesia and the tumor was completely removed via laryngosurgery accompanied by left superior thyroid artery ligation. The specimen was histopathologically compatible with laryngeal angioleiomyoma. Conclusion: Angioleiomyoma of larynx is of benign nature and rarely recurs. However, it can be complicated by profuse bleeding on biopsy and contrast-medium imaging should be considered before surgical intervention to diagnose the tumor properly and prevent life-threatening complications.

Keywords: Laryngeal Angioleiomyoma; Contrast-Enhanced Computed Tomography

\section{INTRODUCTION}

Angioleiomyoma (vascular leiomyoma) is a benign tumor of vascular smooth muscle and occurs frequently in the lower limbs. It is rarely seen in larynx and its recurrence after excision is uncommon. This tumor is presented by hoarseness or pain and the therapy of choice is complete surgical excision. We report a very rare case of angioleiomyoma in 52-year-old Caucasian female with progressive symptom of midnight snoring.

\section{CASE PRESENTATION}

A 52-year-old female complaining about severe midnight snoring, which was bothering her spouse and made him to wake up, was referred to our institute in August, 2010. The patient had also a history of midnight sudden awake and dyspnea. Symptoms were progressive and began from two years ago. She had no history of hoarseness, laryngeal pain, and prior diseases of larynx. Her family and medical histories were unremarkable.

Laryngoscopic findings revealed a large $(30 \times 24 \times 20$ $\mathrm{mm}$ ) well-defined pink tumor with smooth surface originated from the left aryepiglotic fold and covered by normal laryngeal mucosa and no ulceration (Figure 1).

Computed tomography (CT) of larynx demonstrated a soft tissue mass on the left aryepiglotic fold with bulging into the left piriformis sinus (Figure 2), which was severely enhanced in arterial phase of contrast-enhanced CT (Figure 3). In curved multiplanar reconstruction (MPR) images, a small branch of left external carotid artery (ECA) perfused the mass and, therefore, a selective angiography of left ECA was also performed.

The patient visited another hospital and an ear-nosethroat (ENT) surgeon performed a biopsy which was complicated by profuse bleeding of tumor and conventional approaches were not useful in hemorrhage control. 


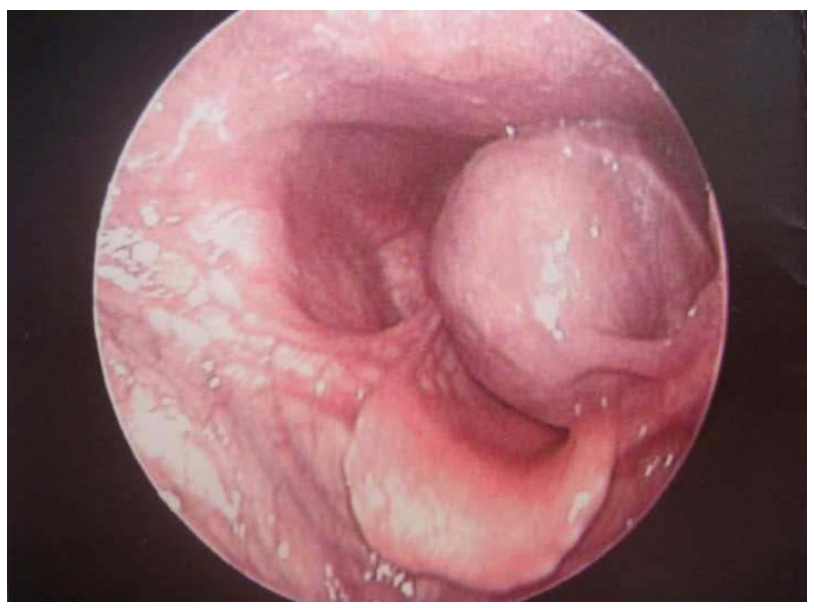

Figure 1. Laryngoscopic view showing a large well-defined mass without any surface ulceration or discoloration, locating on the left aryepiglotic fold. Note the normal laryngeal mucosa.

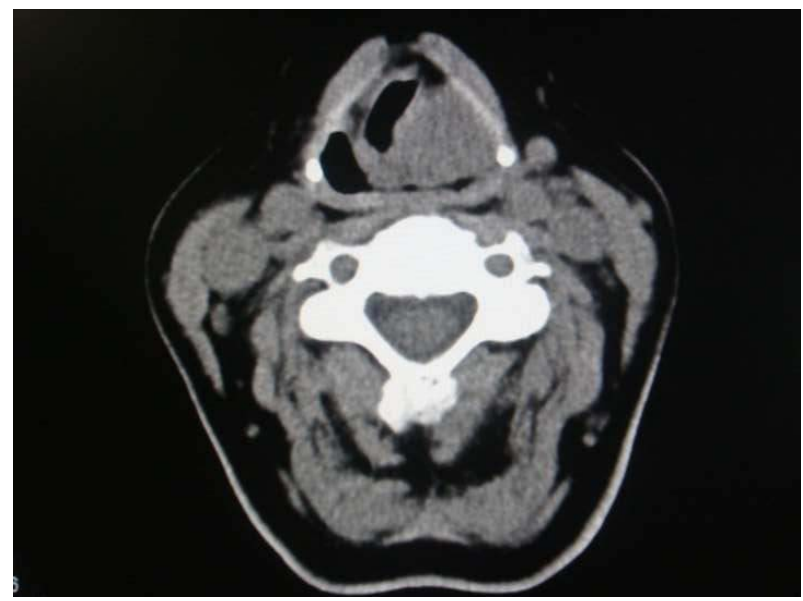

Figure 2. Axial non-enhanced computed tomography of larynx. It shows a large mass of left aryepiglotic fold at the thyroid cartilage level which compromised the laryngeal airway and bulged into the same side piriformis sinus.

Hence, she was administered a general anesthesia and the left superior thyroid artery of external carotid artery was ligated, followed by complete excision of the tumor. Microscopic study showed different size vascular channels surrounded by bundles of smooth muscle cells consistent with angioleiomyoma (Figure 4) and verified positively by immunohistochemical staining for smooth muscle actin (sm-Actin) and endothelial cell antigen CD31 (Figure 5).

She was also investigated for other probable sites of involvement and both physical examination of whole body and sonography of thyroid gland and uterine were normal. Furthermore, laboratory tests showed normal level of parathyroid hormone (PTH: $77.3 \mathrm{pg} / \mathrm{mL}$, normal range: 7 - $82 \mathrm{pg} / \mathrm{mL}$ ).

Her symptoms improved after surgery and no recurrence was occurred after one year of follow-up.

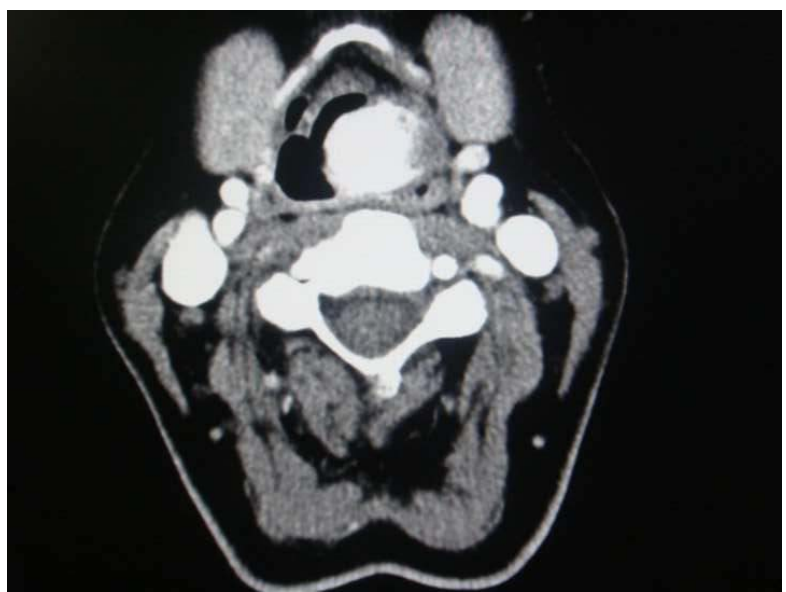

(a)

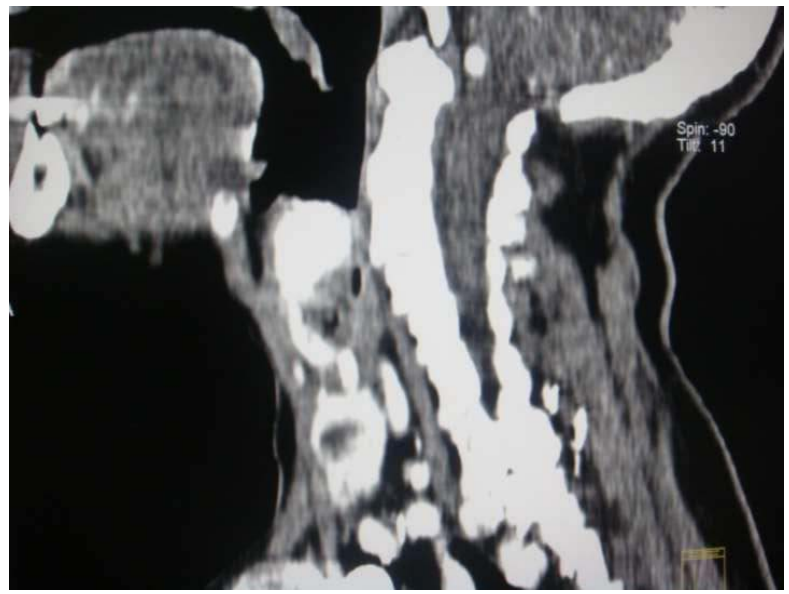

(b)

Figure 3. Contrast-enhanced computed tomography of larynx. Axial view demonstrates a large homogenous enhanced lesion in arterial phase (a). Oblique-sagittal reformat of the contrastenhanced CT confirms vascular supplement of the lesion (b).

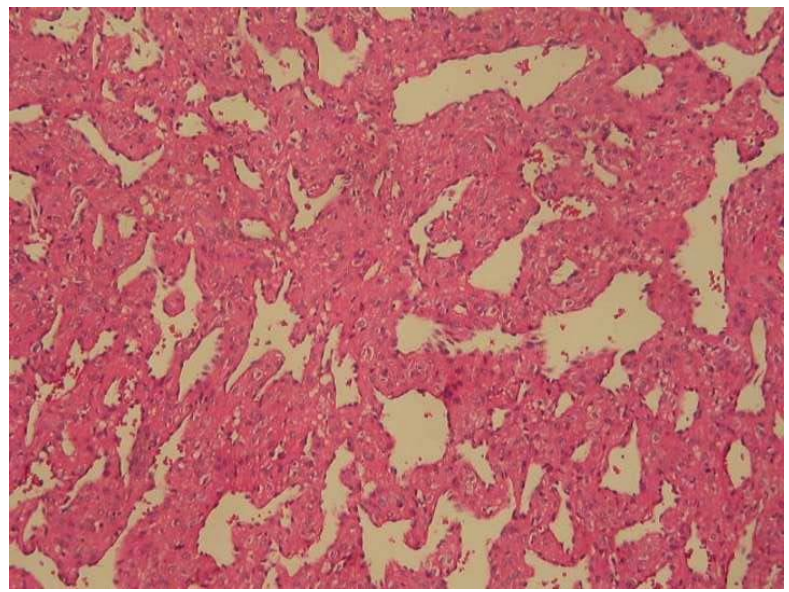

Figure 4. Microscopic view of the laryngeal tumor. It consists of variable size vascular channels that are surrounded by bundles of smooth muscle cells (Hematoxylin-eosin stain, original magnification $\times 250$ ). Note the neoplastic smooth muscle cells clearly related to vascular walls. 


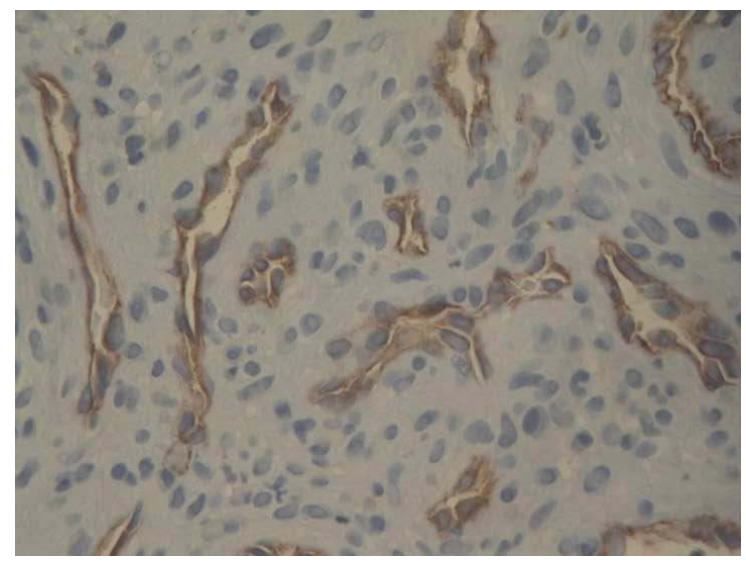

(a)

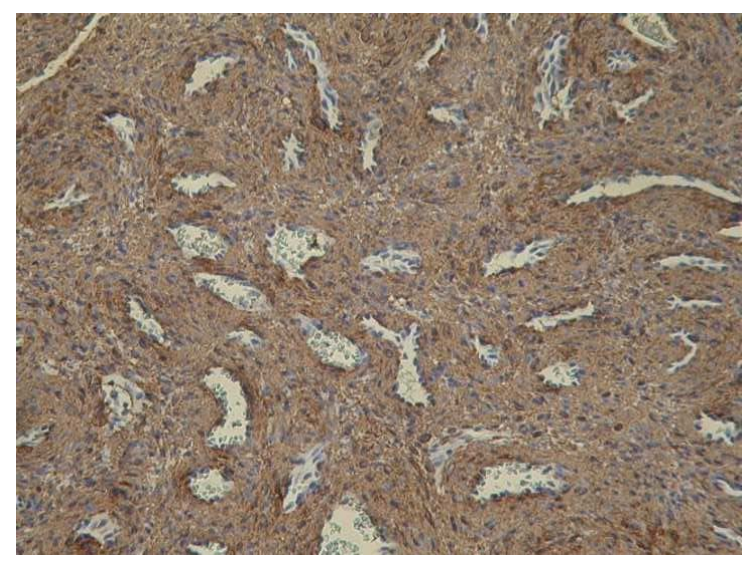

(b)

Figure 5. Immunohistochemical staining for smooth muscle actin. It reveals intimate relationship of neoplastic smooth muscle fibers with vessel walls (original magnification $\times 400(a)$ ). Immunostaining for CD31 highlights endothelial lining of the vascular channels (original magnification $\times 250($ b)).

\section{DISCUSSION}

Angioleiomyomas are benign proliferation of vascular smooth muscles [1] and most often are single and small, but may be seen as a giant multiple spherical tumors [2]. These tumors most commonly involve extremities [3], and do not arise as frequently in the head and neck region [4]. Malignant transformation of these tumors is very rare and it is not reported in laryngeal tumors.

The most common presentation of these tumors in the larynx is hoarseness or dyspnea [5], however, our case was presented with a new symptom-midnight snoring. In addition, most of the angioleiomyoma cases are middleaged and elderly men [1,3], but our case was a female.

The origin and pathogenesis of tumor is still uncertain $[3,6]$. Although regression or atrophy of benign smooth muscle tumors of uterus following the menopause suggests estrogens role in such tumors, it has etiologically little influence on leiomyomas of other organs [7].
Histopathologically, these tumors are classified into three types: solid or capillary, venous, and cavernous [8]. Smooth muscle bundles are closely compacted in solid tumors and intersect with each other. Vascular channels are usually multiple and small-sized. In venous type tumors, smooth muscle bundles are not closely compacted and have venous type vascular channels with thick muscular walls. Dilated vascular channels are seen in cavernous type of these tumors and it is difficult to distinguish muscular walls of these vessels from intervening smooth muscle bundles. Venous type is mainly seen in the head and neck [4]. These tumors can frequently be observed in supraglottic, glottic, and subglottic regions, respectively [3]. Our case was also venous type of angioleiomyoma occurred in supraglotic region of larynx.

Recurrences have been reported after excision in some literatures $[1,8,9]$. Therefore, it has been recommended to monitor patients for recurrence after surgery [3]. We followed the patient for one year and recurrence was not occurred.

It is recommended to avoid biopsy if these tumors are suspected, and CT angiography or MRI should be performed prior to operation [3]. Imaging findings are not the same in different patients. CT features consist of homogenous soft tissue mass with homogenous contrast enhancement. Regarding MRI, these tumors are most commonly isointense on T1-wighted images, heterogenous high signal intense on T2-weighted images, and have a homogenous strong enhancement [10].

Bleeding is a reported complication of these tumors prior or during surgery [11]. The uses of $\mathrm{CO}_{2}$ laser [1] or cryotherapy [5] were reported in initial control of profuse bleeding. These modalities were not available in our institute, and we did surgical incision of larynx and ligation of external carotid artery to control bleeding.

Surgical excision is the treatment of choice and most of the patients need urgent tracheotomy owing to large size of tumor [1]. Direct laryngoscopic or open surgical resection of tumor is determined by tumor size, amount of blood loss, and patient's comorbidities [9]. Direct laryngoscopic resection using laser techniques is now preferred due to its advancement and rapid recovery after resection [12].

\section{CONCLUSION}

Although angioleiomyoma is a benign tumor, it can be complicated by profuse bleeding. Thus, this tumor should be ruled out in conditions that are compatible with vascular tumors and contrast-enhanced imaging such as computed tomography should be performed before laryngoscopic or surgical interventions to prevent serious complications. 


\section{REFERENCES}

[1] Anderson, T.D. and Weinstein, G.S. (2000) Recurrent angiomyoma (vascular leiomyoma) of the larynx after laser excision. Otolaryngology—Head and Neck Surgery, 123, 646-647. doi:10.1067/mhn.2000.110105

[2] Jun, F., Li, L., Ning, L., Yan, L., Siquan, T. and Shixi, L. (2009) Giant laryngeal angioleiomyomas: A case report with review of literature. American Journal of Otolaryngology, 30, 219-220. doi:10.1016/j.amjoto.2008.11.001

[3] Xu, Y., Zhou, S. and Wang, S. (2008) Vascular leiomyoma of the larynx: A rare entity. Three case reports and literature review. Journal for Oto-Rhino-Laryngology and Its Related Specialties, 70, 264-267. doi:10.1159/000133652

[4] Hachisuga, T., Hashimoto, H. and Enjoji, M. (1984) Angioleiomyoma. A clinicopathologic reappraisal of 562 cases. Cancer, 54, 126-130. doi:10.1002/1097-0142(19840701)54:1<126::AID-CNC R2820540125>3.0.CO;2-F

[5] Nuutinen, J. and Syrjänen, K. (1983) Angioleiomyoma of the larynx. Report of a case and review of the literature. Laryngoscope, 93, 941-943. doi:10.1288/00005537-198307000-00019

[6] Farman, A.G. (1975) Benign smooth muscle tumours. South African Medical Journal, 49, 1333-1340.

[7] Hirakawa, K., Harada, Y., Tatsukawa, T., Nagasawa, A. and Fujii, M. (1994) A case of vascular leiomyoma of the larynx. The Journal of Laryngology \& Otology, 108, 593595. doi:10.1017/S0022215100127537

[8] Wang, C.P., Chang, Y.L. and Sheen, T.S. (2004) Vascular leiomyoma of the head and neck. Laryngoscope, 114, 661-665. doi:10.1097/00005537-200404000-00012

[9] Hirshoren, N., Weinberger, J.M., Neuman, T., Ilan, O. and Ben-Yaakov, A. (2010) Recurrent vascular leiomyoma of the larynx: clinical and histopathologic characteristics and treatment. Ear, Nose \& Throat Journal, 89, 382-386.

[10] Yoo, H.J., Choi, J.A., Chung, J.H., Oh, J.H., Lee, G.K., Choi, J.Y., et al. (2009) Angioleiomyoma in soft tissue of extremities: MRI findings. American Journal of Roentgenology, 192, W291-294. doi:10.2214/AJR.07.3952

[11] Shibata, K. and Komune, S. (1980) Laryngeal angiomyoma (vascular leiomyoma): Clinicopathological findings. Laryngoscope, 90, 1880-1886. doi:10.1288/00005537-198011000-00017

[12] Jäckel, M.C., Martin, A. and Steiner, W. (2007) Twentyfive years' experience with laser surgery for head and neck tumors: Report of an international symposium, Göttingen, Germany, 2005. European Archives of Oto-RhinoLaryngology, 264, 577-585. doi:10.1007/s00405-007-0280-6 